Hooyman, G. J.

Mazur, $\mathrm{P}$.

Groot, S. R. de.

1953

\title{
THERMODYNAMICS OF IRREVERSIBLE PROCESSES IN ROTATING SYSTEMS
}

\author{
by G. J. HOOYMAN, H. HOLTAN JR *), P. MAZUR and \\ S. R. DE GROOT **)
}

Instituut voor theoretische natuurkunde, Universiteit, Utrecht, Nederland

\section{Synopsis}

The general entropy balance equation for a rotating system containing a mixture of (charged and uncharged) components is calculated and the influence of centrifugal force and Coriolis force on the entropy production is considered.

From the general formalism the equations for mechanical equilibrium are derived. As a further specification of this case, S v e d b e r g's equations for the determination of molecular masses of uncharged particles are clerived, viz., the equation for the sedimentation equilibrium and (introducing the boundary condition of vanishing volume flow) the equation for the sedimentation rate in the ultracentrifuge. A proper definition of the diffusion coefficient is given.

$\$ 1$. Introduction. In a recent paper $\mathrm{De} G \mathrm{G}$ o o $\mathrm{t}, \mathrm{M}$ a $\mathrm{z} \mathrm{ur}$ and $\mathrm{O} v$ e $\mathrm{b} \mathrm{e} \mathrm{e}^{1}$ ) have discussed the theory of rotating systems and studied the sedimentation potential and the electrophoresis from the point of view of the thermodynamics of irreversible processes $\left.{ }^{2}\right)^{3}$ ). For this case several simplifications could be made. Thus, concentration and temperature gradients were neglected.

In this paper we want to discuss some aspects of the theory without making the assumptions quoted above. In particular, we want to include concentration gradients, upon the formation of which the determination of molecular masses in the ultracentrifuge is based. Detailed considerations concerning temperature gradients will not be given since in actual experiments they are avoided by all

*) Actual address: Det Norske Zinkkompani A/S, Eitrheim, pr. Odda, Norge.

**) Actual address: Instituut voor theoretische natuurkunde, Iniversiteit, Leiden, Nederland. 
available means, as they would cause convection currents and so invalidate the results in the centrifuge. We will therefore only give the basic equations for this case even though the theory of such systems should logically follow as an extension of the theory of thermal diffusion ${ }^{3}$ ), taking the centrifugal force into account.

$\S 2$. The fundamental equations. We consider a system rotating with a constant angular velocity $\boldsymbol{\omega}$. Fixed to the system we choose a right-handed cartesian coordinate system. The state variables of the system are supposed to be continuous functions of space coordinates and of time. The system is composed of $n$ non-reacting components (labelled $k=1,2, \ldots, n$ ), $m$ of which carry electrical charges $e_{k}$ per unit mass $(k=1,2, \ldots, m)$ whereas $e_{m+1}=\ldots=e_{n}=0$. The entropy balance is derived from the four following fundamental equations (see also $\left.\left.{ }^{2}\right)^{3}\right)$ ).

I. The law of conservation of mass. If $\varrho_{k}$ is the density of component $k$ (mass per unit volume), $\partial / \partial t$ the local time derivative and $\mathbf{v}_{k}$ the velocity of substance $k$, this law can be written

$$
\partial \varrho_{k} / \partial t=-\operatorname{div} \varrho_{k} \mathbf{v}_{k}
$$

Introducing the total density

$$
\varrho=\Sigma_{k} \varrho_{k}=1 / v
$$

(with $v$ the specific volume) and the centre of mass velocity

$$
\mathbf{v}=\left(\Sigma_{k} \varrho_{k} \mathbf{v}_{k}\right) / \varrho,
$$

and with the help of the barycentric substantial time derivative

$$
\mathrm{d} / \mathrm{d} t=\partial / \partial t+\mathbf{v} \cdot \operatorname{grad}
$$

and the flow of substance $k$ with respect to $\mathbf{v}$

$$
\mathbf{J}_{k}=\varrho_{k}\left(\mathbf{v}_{k}-\mathbf{v}\right),
$$

onc can write the balance equation ( 1 ) in the alternative form

$$
\operatorname{d} \varrho_{k} / \mathrm{d} t=-\varrho_{k} \operatorname{div} \mathbf{v}-\operatorname{div} \mathbf{J}_{k} .
$$

From (3) and (5) it follows that

$$
\sum_{1}^{n} \mathrm{~J}_{k}=0
$$

Summed over all substances $k$ equation (6) thus leads to

$$
\mathrm{d} \varrho / \mathrm{d} t=-v^{-2} \mathrm{~d} v / \mathrm{d} t=-\varrho \operatorname{div} \mathbf{v}
$$


and finally, with $c_{k}=\varrho_{k} / \varrho$ the mass fraction of $k$, to

$$
\varrho \mathrm{d} c_{k} / \mathrm{d} t=-\operatorname{div} \mathbf{J}_{k} \text {. }
$$

II. Th e f or c e la w (conservation of momentum) reads in the present case if viscous forces are not taken into account

$$
\varrho \mathrm{d} \mathbf{v} / \mathrm{d} t=-\operatorname{grad} P+\sum_{i}^{n} \varrho_{k} \mathbf{F}_{k},
$$

where $P$ is the pressure and $\mathbf{F}_{k}$ the external force on component $k$ per unit mass, consisting of the centrifugal force, the Coriolis force and the electric force

$$
\mathbf{F}_{k}=\omega^{2} \mathbf{r}+2 \mathbf{v}_{k} \wedge \boldsymbol{\omega}+e_{k} \mathbf{E} .
$$

Here $\mathbf{r}$ is the distance from the axis of rotation, $\mathbf{E}$ the electric field strength and $\wedge$ stands for vectorial multiplication.

III. The law of conservation of energy reads

$$
\varrho \mathrm{d}\left(\frac{1}{2} \mathbf{v}^{2}+u\right) / \mathrm{d} t=-\operatorname{div}\left(P \mathbf{v}+\mathbf{J}_{q}\right)+\Sigma_{k} \varrho_{k} \mathbf{F}_{k} \cdot \mathbf{v}_{k} .
$$

Here $u$ is the internal specific energy and $\mathbf{J}_{q}$ the heat flow given by

$$
-\operatorname{div} \mathbf{J}_{q}=\varrho \mathrm{d} q / \mathrm{d} t,
$$

where $\mathrm{d} q$ is the heat supplied to the unit of mass of the mixture.

IV. The second law (Gibbs' equation) is

$$
T \mathrm{~d} s / \mathrm{d} t=\mathrm{d} u / \mathrm{d} t+P \mathrm{~d} v / \mathrm{d} t-\Sigma_{k} \mu_{k} \mathrm{~d} c_{k} / \mathrm{d} t,
$$

where $T$ is the temperature, $s$ the specific entropy and $\mu_{k}$ the chemical potential (partial specific Gibbs function) of $k$.

\$3. The entropy balance. This equation follows from (8), (9), (10), (12), (14) and can be written as

$$
\varrho \mathrm{d} s / \mathrm{d} t=-\operatorname{div} \mathbf{J}_{s}+\sigma,
$$

where

with

$$
\begin{gathered}
T \mathbf{J}_{s}=\mathbf{J}_{q}-\Sigma_{k} \mu_{k} \mathbf{J}_{k}, \\
T \sigma=\mathbf{J}_{q} \cdot \mathbf{X}_{q}+\Sigma_{k} \mathbf{J}_{k} \cdot \mathbf{X}_{k} \geq 0,
\end{gathered}
$$

$$
\begin{gathered}
\mathbf{X}_{q}=-(\operatorname{grad} T) / T, \\
\mathbf{X}_{k}=\mathbf{F}_{k}^{\eta}-T \operatorname{grad}\left(\mu_{k} / T\right) .
\end{gathered}
$$

Equation (15) has the form of a balance equation in which $\mathbf{J}_{s}$ is the flow of entropy and $\sigma$ is the source strength of the entropy production caused by the irreversible processes. In (17) $T \sigma$ is seen to be 
a sum of products of "fluxes" $\mathbf{J}_{q}$ and $\mathbf{J}_{k}$ and of "forces" $\mathbf{X}_{q}$ and $\mathbf{X}_{k}$. In thermodynamics of irreversible processes linear relationships between the components of the fluxes and forces are introduced. To these relations, which are called phenomenological equations, we shall return in subsequent sections.

§ 4. The centrifugal force, the Coriolis force and the entropy production. From (11) and (19) the explicit contributions of the centrifugal force and the Coriolis force to the entropy production (17) can be calculated. The first is $\omega^{2} \mathbf{r} \cdot \Sigma_{k} \mathbf{J}_{k}$ which vanishes according to (7). The second is

$$
\begin{aligned}
2 \Sigma_{k} \mathbf{J}_{k} \cdot \mathbf{v}_{k} \wedge \boldsymbol{\omega} & =2 \Sigma_{k} \varrho_{k} \mathbf{v}_{k} \cdot \mathbf{v}_{k} \wedge \boldsymbol{\omega}-2 \Sigma_{k} \varrho_{k} \mathbf{v} \cdot \mathbf{v}_{k} \wedge \boldsymbol{\omega}= \\
& =0+2 \Sigma_{k} \varrho_{k} \mathbf{v}_{k} \cdot \mathbf{v} \wedge \boldsymbol{\omega}=2 \varrho \mathbf{v} \cdot \mathbf{v} \wedge \boldsymbol{\omega}=0
\end{aligned}
$$

where (3) and (5) have been used. It is thus formally proved that neither the centrifugal force nor the Coriolis force gives any explicit contribution to the entropy production (this reversible character is as is to be expected for motion under the influence of a purely mechanical force).

However, from this result it cannot be concluded that these forces do not give any contribution to the entropy production at all. To make this clear we rewrite $T \sigma$ from (17) with the help of (11), using also (20), and, choosing $T, P$ and the $c_{k}$ as state variables, we express grad $\mu_{k}$ in the gradients of these variables

$T \sigma=\left\{\mathbf{J}_{q}-\Sigma_{k} h_{k} \mathbf{J}_{k}\right\} \cdot \mathbf{X}_{q}+\Sigma_{k} \mathbf{J}_{k} \cdot\left\{e_{k} \mathbf{E}-v_{k} \operatorname{grad} \mathrm{P}-\left(\operatorname{grad} \mu_{k}\right)_{P, T}\right\},(21)$

where $h_{k}$ and $v_{k}$ are the partial specific enthalpy and volume of $k$, respectively. For the case of mechanical equilibrium $(\mathrm{d} \mathbf{v} / \mathrm{d} t=0)$, which is of particular interest, the force law (10) shows the connection between grad $P$ and the external forces. It is then seen that in this case these forces contribute implicitly to $T \sigma$, because they give rise to a pressure gradient which appears in the expression (21).

$\S 5$. The case of mechanical equilibrium and the condition of vanishing volume flow. The setting up of mechanical equilibrium is a very much quicker process than any thermodynamical phenomenon. It will virtually be reached as soon as $\omega$ has attained a constant value. We first want to consider this case without making any further approximation. 
A. Mechanical equilibrium. In this case, where $\mathrm{d} \mathbf{v} / \mathrm{d} t=0$, we can apply a theorem which is due to $\left.\left.\mathrm{Prigog} \mathrm{in}{ }^{2}\right)^{3}\right)$. In order to do this in the general non-isothermal case we perform a linear transformation of the fluxes and forces in (17) leaving $T \sigma$ invariant

$$
T \sigma=\mathbf{J}_{q}^{\prime} \cdot \mathbf{X}_{q}^{\prime}+\sum_{1}^{\prime \prime} \mathbf{J}_{k}^{\prime} \cdot \mathbf{X}_{k}^{\prime}
$$

If we choose as new fluxes

$$
\begin{gathered}
\mathbf{J}_{q}^{\prime}-\mathbf{J}_{q}-\Sigma_{k} h_{k} \mathbf{J}_{k}, \\
\mathbf{J}_{k}^{\prime}=\mathbf{J}_{k},
\end{gathered}
$$

we find, identifying (17) and (22)

$$
\begin{gathered}
\mathbf{X}_{q}^{\prime}=\mathbf{X}_{q}=-(\operatorname{grad} T) / T \\
\mathbf{X}_{k}^{\prime}=\mathbf{X}_{k}+h_{k} \mathbf{X}_{q}=\mathbf{F}_{k}-\left(\operatorname{grac}_{k} \mu_{k}\right.
\end{gathered}
$$

Prigogine has shown that, assuming mechanical equilibrium and using forces and fluxes of the form (23)-(26) (with $\mathbf{F}_{k}$ the force from the force law (10)), one can replace the barycentric velocity $\mathbf{v}$ in the flows $\mathbf{J}_{k}^{\prime}$ appearing in (22) by an arbitrary other velocity.

Let us introduce an arbitrary mean velocity

$$
\mathbf{v}^{a} \equiv \sum_{1}^{n} w_{k} \mathbf{v}_{k}
$$

where the $w_{k}$ are weight factors, normalized to unity

$$
\Sigma_{k} w_{k}=1 \text {. }
$$

With this velocity we can define the flows

for which

$$
\mathbf{J}_{k}^{a} \equiv \varrho_{k}\left(\mathbf{v}_{k}-\mathbf{v}^{a}\right)
$$

holds.

$$
\sum_{1}^{n} w_{k} \mathbf{J}_{k}^{a} / \varrho_{k}=0
$$

The use of $\mathbf{J}_{k}^{a}$ instead of $\mathbf{J}_{k}^{\prime}$ in (22) leads to

$$
\begin{array}{r}
T \sigma=\mathbf{J}_{q}^{\prime} \cdot \mathbf{X}_{q}^{\prime}+\sum_{1}^{n} \mathbf{J}_{k}^{a} \cdot\left\{\left(1-\varrho v_{k}\right)\left(\omega^{2} \mathbf{r}+2 \mathbf{v} \wedge \boldsymbol{\omega}\right)+\left(e_{k}-\varrho v_{k} e\right) \mathbf{E}-\right. \\
\left.-\left(\operatorname{grad} \mu_{k}\right)_{P^{\prime}, T}\right\}
\end{array}
$$

where we have applied

$\operatorname{grad} \mu_{k}=-s_{k} \operatorname{grad} T+v_{k} \operatorname{grad} P+\sum_{i}^{n-1}\left(\partial_{\mu_{k}} / \partial c_{i}\right)_{P, T} \operatorname{grad} c_{i}$, (32) $\left(s_{k}\right.$ being the partial specific entropy of component $k$ ) and the condition of mechanical equilibrium,

$$
\operatorname{grad} P=\varrho \omega^{2} \mathbf{r}+2 \varrho \mathbf{v} \wedge \omega+\varrho e \mathbf{E},
$$


derived from (10) and (11); we also have used the equality

$$
\begin{aligned}
\Sigma_{k} \mathbf{J}_{k}^{a} \cdot \mathbf{v}_{k} \wedge \boldsymbol{\omega}=\Sigma_{k} \mathbf{J}_{k}^{a} \cdot \mathbf{v}^{a} & \wedge \boldsymbol{\omega}=\varrho\left(\mathbf{v}-\mathbf{v}^{a}\right) \cdot \mathbf{v}^{a} \wedge \boldsymbol{\omega}= \\
& =\varrho\left(\mathbf{v}-\mathbf{v}^{a}\right) \cdot \mathbf{v} \wedge \boldsymbol{\omega}=\Sigma_{k} \mathbf{J}_{k}^{a} \cdot \mathbf{v} \wedge \boldsymbol{\omega} .
\end{aligned}
$$

Finally, $e$ stands for the total charge per unit mass

$$
e=\Sigma_{k} c_{k} e_{k} \text {. }
$$

Let us consider some special choices of $\mathbf{v}^{a}$ and the relative flows $\left.\left.\left(\text { see also }{ }^{3}\right)^{4}\right)\right)$ :

a) Barycentric velocity, $w_{k}=c_{k}$. This leads to the centre of mass velocity $\mathbf{v}$ defined already in (3) and to the barycentric description used in the foregoing sections.

b) M e a n molar velocity, $w_{k}=n_{k}$. With $M_{k}$ the molar mass of substance $k, N_{k}=\varrho_{k} / M_{k}$ the molar concentration, $N=$ $\Sigma_{k} N_{k}$ the molar density and $n_{k}=N_{k} / N$ the mole fraction of $k$, the mean molar velocity is

$$
\mathbf{v}^{m}=\Sigma_{k} n_{k} \mathbf{v}_{k}
$$

With this velocity it is sometimes of advantage to use molar flows instead of mass flows, i.e.

$$
\mathbf{J}_{k, m o l}^{m} \equiv N_{k}\left(\mathbf{v}_{k}-\mathbf{v}^{m}\right)=\mathbf{J}_{k}^{m} / M_{k},
$$

for which, of course,

$$
\Sigma_{k} \mathbf{J}_{k, m o l}^{m}=0
$$

holds, analogous to (7).

c) Mean volume velocity, $w_{k}=\varrho_{k} v_{k}$. Here we have

and

$$
\mathbf{v}^{\circ}=\Sigma_{k} \varrho v_{k} \mathbf{v}_{k} \text {, }
$$

$$
\Sigma_{k} \varrho_{k} v_{k}=1
$$

d) Velocity of component $i, w_{i}=1, w_{k}=0(k \neq i)$. The use of material flows with respect to one of the components is of special interest for dilute solutions where one can take the solvent for component $i$.

The use of Prig o g i n e's theorem is not restricted to the introduction of mean velocities (27). One also could choose a constant velocity, e.g. $\mathbf{v}^{a}=0$, thus passing on to absolute flows $\varrho_{k} \mathbf{v}_{k}$. For this choice, equation (31) still holds. 
$B$. Vanishing volume flow. From (31) it is seen that at mechanical equilibrium, the entropy production $T \sigma$ contains contributions of the centrifugal force

$$
\Sigma_{k} \mathbf{J}_{k}^{a} \cdot\left(1-\varrho v_{k}\right) \omega^{2} \mathbf{r}=\varrho\left(\mathbf{v}-\mathbf{v}^{\circ}\right) \cdot \omega^{2} \mathbf{r},
$$

of the Coriolis force

$$
2 \Sigma_{k} \mathbf{J}_{k}^{a} \cdot\left(1-\varrho v_{k}\right) \mathbf{v} \wedge \omega=2 \varrho \mathbf{v} \cdot \mathbf{v}^{\circ} \wedge \omega,
$$

and of the electrical phenomena

$$
\Sigma_{k} \mathbf{J}_{k}^{a} \cdot\left(e_{k}-\varrho v_{k} e\right) \mathbf{E}=\left(\mathbf{I}-\varrho e \mathbf{v}^{\circ}\right) \cdot \mathbf{E},
$$

with $\mathbf{v}^{\circ}$ the mean volume velocity (39), and I the electrical current

$$
\mathbf{I}=\Sigma_{k} \varrho_{k} e_{k} \mathbf{v}_{k} \text {. }
$$

As a first approximation, one could neglect all transverse effects, thus neglecting the Coriolis force with respect to the centrifugal force. This is justified since $\left|\mathbf{v}_{k}\right| \ll \omega r$. In practical cases, the boundary condition for a liquid confined to a vessel can then be expressed by the vanishing of the total volume flow through any section (cf. Appendix I) or

$$
\mathbf{v}^{\circ}=0 \text {. }
$$

If, moreover, the gradients of temperature and concentrations could be neglected, (31) would reduce to

$$
T \sigma=\mathbf{J} \cdot \omega^{2} \mathbf{r}+\mathbf{I} \cdot \mathbf{E},
$$

with the total mass flow

$$
\mathbf{J}=\varrho \mathbf{v}=\Sigma_{k} \varrho_{k} \mathbf{v}_{k},
$$

or, eliminating the absolute flow $\varrho_{n} \mathbf{v}_{n}$ by means of (39) and (45), to

$$
T \sigma=\Sigma_{1}^{n-1} \varrho_{k} \mathbf{v}_{k} \cdot\left\{e_{k} \mathbf{E}+\left(1-v_{k} / v_{n}\right) \omega^{2} \mathbf{r}\right\},
$$

which were the basic equations for the treatment of the sedimentation potential and electrophoresis given by $\mathrm{De} G \mathrm{root}, \mathrm{Maz} \mathrm{ur}$

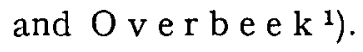

\$6. The sedimentation equilibrium for an uncharged monodisperse system in the ultracentrifuge. The foregoing treatment until $\S 5 B$ is applicable to the particular case of an isothermal solution of one uncharged solute in a solvent, which case is of practical interest for 
determining molecular masses by means of sedimentation in an ultracentrifuge. With $n=2, \operatorname{grad} T=0$ and $e_{1}=e_{2}=0$, equation (31) reads

$$
T \boldsymbol{\sigma}=\mathbf{J}_{1}^{a} \cdot w_{2}^{-1}\left\{\left(1-\varrho v_{1}\right)\left(\omega^{2} \mathbf{r}+2 \mathbf{v} \wedge \boldsymbol{\omega}\right)-\left(\operatorname{grad} \mu_{1}\right)_{P, T}\right\} \geq 0,
$$

where we have eliminated $\mathbf{J}_{2}^{a}$ by means of (30) and made use of the Gibbs-Duhem relation

$$
\sum_{1}^{n} \varrho_{k}\left(\operatorname{grad} \mu_{k}\right)_{P, T}=0
$$

and the identity

$$
\Sigma_{k} o_{k}\left(1-\varrho v_{k}\right)=0 .
$$

According to the general procedure in thermodynamics of irreversible processes ${ }^{2}$ ) ${ }^{3}$ ) we can assume linear phenomenological relations between the fluxes and forces occurring in the entropy production:

$$
\mathbf{J}_{1}^{a}=\mathrm{L} w_{2}^{1}\left\{\left(1-\varrho v_{1}\right)\left(\omega^{2} \mathbf{r}+2 \mathbf{v} \wedge \boldsymbol{\omega}\right)-\left(\operatorname{grad} \mu_{1}\right)_{P, T}\right\} .
$$

Here $L$ is a tensor of the second order.

We first consider thermostatic equilibrium, i.e. the vanishing of the entropy production. Then from (49) and (52) it follows that fluxes and forces also vanish. Therefore, since in that case $\mathbf{v}_{1}=\mathbf{v}_{2}$, which for a closed vessel means that $\mathbf{v}=0$, we have

$$
\left(1-\varrho v_{1}\right) \omega^{2} \mathbf{r}-\left(\operatorname{grad} \mu_{1}\right)_{P, T}=0 .
$$

This equation allows us to describe the distribution of the solute in sedimentation equilibrium with the help of

$$
\begin{aligned}
\left(\operatorname{grad} \mu_{1}\right)_{P, T}=\left(\partial \mu_{1} / \partial n_{1}\right)_{P, T} \operatorname{grad} n_{1} & =\left(\partial \mu_{1} / \partial c_{1}\right)_{P, T} \operatorname{grad} c_{1}= \\
& =\left(\partial \mu_{1} / \partial \varrho_{1}\right)_{P, T} \operatorname{grad} \varrho_{1} .
\end{aligned}
$$

For an ideal solution

$$
\left(\partial \mu_{1} / \partial n_{1}\right)_{P, T}=R T / M_{1} n_{1}
$$

and with the help of

and

$$
\mathrm{d} n_{1} / \mathrm{d} c_{1}=n_{1} n_{2} / c_{1} c_{2}
$$

$$
\left(\partial \varrho_{1} / \partial c_{1}\right)_{P, T}=\varrho^{2} v_{2}
$$

(ct. Appendix II) we find

$$
\begin{gathered}
n_{1}^{-1} \partial n_{1} / \partial r=M_{1}\left(1-\varrho v_{1}\right) \omega^{2} r / R T \\
c_{1}^{-1} \partial c_{1} / \partial r=\left\{1-c_{1}\left(1-M_{2} / M_{1}\right)\right\} M_{1}\left(1-\varrho v_{1}\right) \omega^{2} r / R T \\
\varrho_{1}^{-1} \partial \varrho_{1} / \partial r=N_{1}^{-1} \partial N_{1} / \partial r=M_{1}\left[1-\varrho v_{1}\left\{1+c_{1}\left(1-\varrho v_{1}\right)\right\}\right] \omega^{2} r / R T n_{2}
\end{gathered}
$$


Equation (58) is the well-known $\mathrm{S} v$ e d berg equation (ct. ${ }^{5}$ ) p. 46 , equ. $(91 a)$ ) for the sedimentation equilibrium. In practical applications to the determination of the molecular mass $M_{1}$ the expressions on the left-hand sides of (58)-(60) are taken to be equal. From the above it can be seen which approximations are necessary in order to justify this procedure. In practical cases $M_{2} \ll M_{1}$. Generally the solution will be molar diluted, so $n_{2} \approx 1$. If the solution is so highly diluted that also $c_{2} \approx 1$ the right-hand sides of (59) and $(60)$ reduce to the right-hand side of (58).

§7. The sedimentation velocity for an uncharged monodisperse system. Before considering the sedimentation process we want to give a proper definition of the diffusion coefficient.

$A$. Definition of the diffusion coefficient ${ }^{4}$ ). As can be seen, e.g., from (49), in the case of isothermal diffusion in a binary uncharged system in the absence of external forces the entropy production at mechanical equilibrium (where the pressure $P$ is uniform, cf. (33)) is given by

$$
T \sigma=-\mathbf{J}_{1}^{a} \cdot w_{2}^{-1}\left(\partial \mu_{1} / \partial c_{1}\right)_{P, T} \operatorname{grad} c_{1} .
$$

In general, the phenomenological relation between $\mathbf{J}_{1}^{n}$ and the "force" occurring in (61) will be a tensorial relation. If transverse effects are negligible this relation reduces to a vectorial one for an isotropic fluid and can be written

$$
\mathbf{J}_{1}^{a}=-w_{2} c_{2}^{-1} \varrho D \operatorname{grad} c_{1},
$$

which is $\mathrm{F}$ i c k's law and defines the diffusion coefficient $D$. (In the presence of external forces, the right-hand side of (62) would contain these forces as well, but one still could define $w_{2} c_{2}^{-1} \varrho D$ as the coefficient of $-\operatorname{grad} c_{1}$ ). From the invariance of $T \sigma$ it follows that $D$ is independent of the special choice of the $w_{k}$, which can be seen immediately by inserting (62) in (61). Moreover, the relation (62) is symmetric in the two components: with (30) it follows from (62) that

$$
\mathbf{J}_{2}^{a}=-w_{1} c_{1}^{-1} \varrho D \operatorname{grad} c_{2} .
$$

Finally, $D$ has the same dimensions as a surface area divided by a time ${ }^{*}$ ).

*) The introduction of the flows $J_{k}^{a}$ and of the diffusion coefficient $D$ having the properties stated here is not restricted to the case of mechanical equilibrium or to the absence of a temperature gradient. 
Of course, (62) allows us to express the flow (molar or in mass units) in terms of the gradient of $n_{1}, \varrho_{1}$ or $N_{1}$. One has, e.g.,

$$
\mathbf{J}_{1, m o l}^{a}=-e_{2} n_{2}^{-1} N D \operatorname{grad} n_{1},
$$

or, with the help of (57),

$$
\mathbf{J}_{1}^{a}=-w_{2}\left(\varrho_{2} v_{2}\right)^{-1} D \operatorname{grad} \varrho_{1} .
$$

It is now easy to derive the various forms in which Fic k's law is usually stated. We will give some of them here.

The barycentric description $\left(w_{k}=c_{k}\right)$ of the diffusion phenomenon leads to

$$
\mathbf{J}_{1} \equiv \varrho_{1}\left(\mathbf{v}_{1}-\mathbf{v}\right)=-\varrho D \operatorname{grad} c_{1}
$$

With the molar description $\left(w_{k}=n_{k}\right)$ (64) yiclds

$$
\mathbf{J}_{1, \text { mol }}^{m} \equiv N_{\mathbf{1}}\left(\mathbf{v}_{1}-\mathbf{v}^{m}\right)=-N D \operatorname{grad} n_{1} \text {. }
$$

If one prefers to consider the diffusion with respect to the mean volume velocity $\left(w_{k}=\varrho_{k} v_{k}\right)$, the most convenient relations, fitting in this scheme, are

and

$$
\mathbf{J}_{1}^{\circ} \equiv \varrho_{1}\left(\mathbf{v}_{1}-\mathbf{v}^{\circ}\right)=-D \operatorname{grad} \varrho_{1},
$$

$$
\mathbf{J}_{1, m o l}^{\circ} \equiv N_{1}\left(\mathbf{v}_{1}-\mathbf{v}^{\circ}\right)=-D \operatorname{grad} N_{1} .
$$

Finally, taking relative flows with respect to the velocity of component 2, we have

and

$$
\mathbf{J}_{1}^{r} \equiv \varrho_{1}\left(\mathbf{v}_{1}-\mathbf{v}_{2}\right)=-c_{2}^{-1} \varrho D \operatorname{grad} c_{1}
$$

$$
\mathbf{J}_{1, \text { al }}^{r} \equiv N_{1}\left(\mathbf{v}_{1}-\mathbf{v}_{2}\right)=-n_{2}^{-1} N D \operatorname{grad} n_{1} .
$$

Several authors ${ }^{3}{ }^{4}$ ) have given relations between the diffusion coefficients, occurring in the various descriptions of the diffusion phenomenon. It is the advantage of the definition (62) of $D$ that it immediately connects the various phenomenological coefficients which occur according to the choice of the reference velocity, the kind of flow and the gradient to which this flow is related.

$B$. The sedimentation velocity. Another important method for determining molecular masses in the ultracentrifuge consists in following the sedimentation rate of the layer between solution and pure solvent.

In order to find an expression for this velocity we start again from the relation (52). If we neglect, as we did in $\$ \$ 5 B$ and $7 A$, transverse 
effects, the Coriolis force in (52) can be left out and the tensor $L$ reduces for an isotropic fluid to a scalar $L$. Since by (62) the diffusion coefficient $D$ is defined by taking the coefficient of $-\operatorname{grad} c_{1}$ equal to $w_{2} c_{2}^{-1} \varrho D$, we have

$$
L=w_{2}^{2} \varrho c_{2}^{-1}\left(\partial \mu_{1} / \partial c_{1}\right)_{P, T}^{-1} D,
$$

or for an ideal solution

$$
L=w_{2}^{2} \varrho_{1} M_{1} D / R T n_{2} .
$$

As long as the system is still far from equilibrium the solution is homogeneous over a large region between the boundary (separating solution and pure solvent) and the outer wall, i.e. $\operatorname{grad} c_{1}=0$. Therefore, in this region (52) becomes

$$
\mathbf{J}_{1}^{a}=w_{2} \varrho_{1} M_{1} D\left(1-\varrho v_{1}\right) \omega^{2} \mathbf{r} / R T n_{2} .
$$

Introducing the mobility

$$
U_{1}^{a}=\left|\mathbf{v}_{1}-\mathbf{v}^{a}\right| / M_{1}\left(1-\varrho v_{1}\right) \omega^{2} r,
$$

we can write equ. (74), using also (29),

$$
U_{1}^{u}=w_{2} D / R T n_{2},
$$

which can be considered as a generalization of the Einstein relation between diffusion coefficient and mobility. With the molar description $\left(w_{k}=n_{k}\right)$ it takes the form

$$
U_{1}^{m}=D / R T \text {. }
$$

We now can consider the flow (74) with respect to the mean volume velocity $\mathbf{v}^{\circ}$, which can be taken zero according to the boundary condition (45). We thus have from (29)

or *)

$$
\mathbf{v}_{1}=\varrho_{2} v_{2} M_{1} D\left(1-\varrho v_{1}\right) \omega^{2} \mathbf{r} / R T n_{2},
$$

$$
M_{1}=n_{2} R T\left|\mathbf{v}_{1}\right| /\left[1-\varrho v_{1}\left\{1+c_{1}\left(1-\varrho v_{1}\right)\right\}\right] D \omega^{2} r .
$$

In the same way as in $\S 6$ one can make the approximation $n_{2} \approx 1$ or the more restrictive approximation $c_{2} \approx 1$. The latter one would lead to the well-known $\mathrm{S}$ ve d be $\mathrm{r} g$ equation

$$
M_{1}=R T\left|\mathbf{v}_{1}\right| /\left(1-\varrho v_{1}\right) D \omega^{2} r .
$$

The equations (78)-(80) are valid throughout the region where

*) The above treatment also holds for a non-ideal solution if $R T$ is replaced by $\left(\partial \mu_{1}+/ \partial \ln n_{1}\right)_{P, T}$, where $\mu_{1} t$ is the partial molar Gibbs function. The formulae thus obtained correspond with those given by $\mathrm{L} \mathrm{a} \mathrm{m} \mathrm{m}^{6}$ ).

Physica XIX 
grad $c_{1}$ is negligible. In practice one measures $\left|\mathbf{v}_{1}\right|$ by observing a part of the boundary layer of which the motion also follows from (78)-(80).

The $\mathrm{S} v e \mathrm{dberg}$ equations have thus been derived in the framework of thermodynamics of irreversible processes which allows a rigorous definition of all quantities involved. Thus we have found the result (79) and as an approximation the customary relation (80), both containing well-defined quantities $\left(D\right.$ and $\left.\varrho v_{1}\right)$. In this way the non-equilibrium equation has been put on a basis which meets the same requirements of exactness as the equilibrium equation ${ }^{7}$ ).

\section{APPENDIX I}

The condition of vanishing volume flow ${ }^{1}$ ). The mean volume velocity $\mathbf{v}^{\circ}$, defined in (39), can be shown to be zero if one can neglect transverse effects and if the partial specific volumes $\mathbf{v}_{k}$ do not apprcciably depend on pressure and concentrations.

From the identity (40) it follows that

$$
\Sigma_{k} v_{k} \partial \varrho_{k} / \partial t+\Sigma_{k} \varrho_{k} \partial v_{k} / \partial t=0
$$

With the help of this identity and the conservation of mass (1) one obtains

$$
\operatorname{div} \mathbf{v}^{\circ}=\Sigma_{k} \varrho_{k}\left(\partial v_{k} / \partial t+\mathbf{v}_{k} \cdot \operatorname{grad} v_{k}\right) .
$$

The expression at the right-hand side in parentheses is seen to be the substantial time derivative of $v_{k}$ in the direction of $\mathbf{v}_{k}$ and is zero according to the above assumption.

The same assumption allows us to write

$$
\operatorname{rot} \mathbf{v}^{\circ}=\Sigma_{k} \operatorname{rot} v_{k} \mathbf{J}_{k}+\operatorname{rot} \mathbf{v}=\Sigma_{k} v_{k} \operatorname{rot} \mathbf{J}_{k}+\operatorname{rot} \mathbf{v} .
$$

As was stated in $\S 3$, linear relations exist between the components of the fluxes and forces occurring in the entropy production. As can be seen from (17), this means that a tensorial relation exists between $\mathbf{J}_{k}$ and the forces $\mathbf{X}_{q}$ and $\mathbf{X}_{i}(i=1, \ldots, n)$ given in (18) and (19). If we then can neglect transverse effects (and in particular disregard the Coriolis force), the isotropy of the fluid reduces this tensorial relation to a vectorial one of the form

and in consequence

$$
\mathbf{J}_{k}=\Sigma_{j} L_{k i} \operatorname{grad} Y_{j}
$$

$$
\operatorname{rot} \mathbf{J}_{k}=\Sigma_{j} \operatorname{grad} L_{k j} \wedge \operatorname{grad} Y_{j} .
$$


This expression vanishes since, in accordance with the neglecting of transverse effects, $\operatorname{grad} L_{k j}$ is parallel to grad $Y_{j}$.

The supposition, made throughout the whole treatment, that viscous phenomena do not give an appreciable contribution to the entropy production, allows us to neglect the quantities $\partial v_{i} / \partial x_{j}+$ $+\partial v_{j} / \partial x_{i}(i, j$ cartesian coordinates). Since the tangential component of $\mathbf{v}$ vanishes at the wall, this gives rot $\mathbf{v}=0$.

As a consequence, rot $\mathbf{v}^{\circ}=0$, which combines with $\operatorname{div} \mathbf{v}^{\circ}=.0$ and the fact that the normal component of $\mathbf{v}^{\circ}$ vanishes at the boundary to yield the condition of vanishing volume flow $\mathbf{v}^{\circ}=0$.

\section{APPENDIX II}

Equation (56) is readily derived from the definition of the mole fractions

$$
n_{i}=\left(c_{i} / M_{i}\right) /\left(c_{1} / M_{1}+c_{2} / M_{2}\right), \quad(i=1,2) .
$$

In order to arrive at equation (57) we consider a homogeneous mixture of $n$ components containing the masses $m_{k}(k=1, \ldots, n)$ in a volume $V$. The partial specific volumes $v_{k}$ are then defined by

$$
v_{k}=\left(\partial V / \partial m_{k}\right)_{P, T, m_{i}} .
$$

Since $V$ is a homogeneous function of the first degree of the $m_{k}$, we have the Euler relation

and hence

$$
V=\sum_{1}^{n} m_{k} v_{k}
$$

$$
\delta V=\sum_{1}^{n} v_{k} \delta m_{k}+\sum_{1}^{n} m_{k} \delta v_{k} .
$$

On the other hand, we have with constant $P$ and $T$

$$
\delta V=\Sigma_{1}^{n}\left(\partial V / \partial m_{k}\right)_{P, T, m_{i}} \delta \dot{m}_{k}=\sum_{1}^{n} v_{k} \delta m_{k},
$$

which combines with (89) to yield for constant $P$ and $T$

$$
\sum_{i}^{n} m_{k} \delta v_{k}=0 .
$$

Introducing the density $\varrho_{k}=m_{k} / V$, we can write equations (88) and (91) in the form

and

$$
1=\sum_{1}^{n} \varrho_{k} v_{k}
$$

$$
\sum_{1}^{n} \varrho_{k} \delta v_{k}=0,
$$


respectively. From these relations it then follows that for constant $P$ and $T$

$$
\sum_{i}^{n} v_{k} \delta \varrho_{k}=0
$$

For a binary system we can take $P, T$ and $c_{1}$ as state variables and (94) leads to

$$
v_{1}\left(\partial \varrho_{1} / \partial c_{1}\right)_{P, T}+v_{2}\left(\partial \varrho_{2} / \partial c_{1}\right)_{P, T}=0
$$

or, writing $\varrho_{2}$ as $\underline{\varrho}_{1}\left(c_{1}^{-1}-1\right)$,

$$
\left(\partial \varrho_{1} / \partial c_{1}\right)_{P, T}=\varrho^{2} v_{2}
$$

Equation (58) follows immediately from (53), (54) and (55). In order to derive (59) one needs $\left(\partial \mu_{1} / \partial c_{1}\right)_{P, T}$ which can be calculated from (55) and (56):

$\left(\partial \mu_{1} / \partial c_{1}\right)_{P, T}=R T n_{2} / M_{1} c_{1} c_{2}-R T / M_{1} c_{1}\left\{1-c_{1}\left(1-M_{2} / M_{1}\right)\right\}$

where the definition (86) of $n_{2}$ has been used. Finally, we can write, taking $P, T$ and $\varrho_{1}$ as state variables,

$$
\delta \mu_{1}=\left(\partial \mu_{1} / \partial \varrho_{1}\right)_{P, T} \delta \varrho_{1},
$$

for constant $P, T$. Passing on to $P, T$ and $c_{1}$ as state variables, we then find that

$$
\left(\partial \mu_{1} / \partial c_{1}\right)_{P, T}=\left(\partial \mu_{1} / \partial \underline{Q}_{1}\right)_{P, T}\left(\partial Q_{1} / \partial c_{1}\right)_{P, T}
$$

which combines with (97) and (57) to give $\left(\partial \mu_{1} / \partial \varrho_{1}\right)_{P, T}$ and so equation (60).

Received 9-9-53.

\section{REFERENCES}

1) Groot, S. R. de, M a z r, P. and Overbeek, J. Th. G., J. chem. Phys. 20 (1952) 1825.

2) Prigogine, I., Ftude thermodynamique des phénomènes irréversibles, Thesis, Brussels, 1947.

3) Groot, S. R. de, Thermodynamics of irreversible processes, North Holland Publishing Company, Amsterdam and Interscience Publishers, New York, 1951.

b) Prig o g i n e, I., Bull. Acad. roy. Belg., Cl. Sc. [5] 34 (1948) 930.

5) Svedberg, The and Pedersen, $k$. O., Die Ultrazentrifuge, Steinkopff, Dresden und Leipzig, 1940.

6) L a m m, O., Acta chem. Scand., 7 (1953) 173.

7) L o n g s w or th, L. G., Proc. nat. Acad. Sc. 36 (1950) 502. 